\title{
Odorant Feature Detection: Activity Mapping of Structure Response Relationships in the Zebrafish Olfactory Bulb
}

\author{
Stefan H. Fuss and Sigrun I. Korsching \\ Institut für Genetik, Universität zu Köln, 50674 Köln, Germany
}

The structural determinants of an odor molecule necessary and/or sufficient for interaction with the cognate olfactory receptor(s) are not known. Olfactory receptor neurons expressing the same olfactory receptor converge in the olfactory bulb. Thus, optical imaging of neuronal activity in the olfactory bulb can visualize at once the contributions by all the different olfactory receptors responsive to a particular odorant. We have used this technique to derive estimates about the structural requirements and minimal number of different zebrafish olfactory receptors that respond to a series of naturally occurring amino acids and some structurally related compounds.

We report that the $\alpha$-carboxyl group, the $\alpha$-amino group, and L-conformation of the amino acid are all required for activation of amino acid-responsive receptors. Increasing carbon chain length recruits successively more receptors. With increasing concentrations, the activity patterns induced by a homolog series of amino acids became more similar to each other. At intermediate concentrations patterns were unique across substances and across concentrations.

The introduction of a terminal amino group (charged) both recruits additional receptors and prevents binding to some of the receptors that were responsive to the unsubstituted analog. In contrast, the introduction of a $\beta$-hydroxyl group (polar) excluded the odorants from some of the receptors that are capable of binding the unsubstituted analog. Cross-adaptation experiments independently confirmed these results. Thus, odorant detection requires several different receptors even for relatively simple odorants such as amino acids, and individual receptors require the presence of some molecular features, the absence of others, and tolerate still other molecular features.

Key words: olfactory bulb; odorant feature; zebrafish; odorant; olfactory receptor; Danio rerio; Calcium Green; optical imaging
Odor space is multidimensional because of the vast multitude of olfactory receptors (ORs) involved in odorant recognition [ 1000 in rat (Buck and Axel, 1991), 100 in fish (Ngai et al., 1993; Barth et al., 1996; Korsching et al., 1997), 57 in Drosophila (Vosshall et al., 2000), 100 in Caenorhabditis elegans (Troemel et al., 1995)]. Each OR can be thought of as spanning a dimension of this odor space. A particular molecule qualifies as odorant if it interacts with at least one of these ORs. The perceived odor of an odorant is determined by the characteristic combination of the OR that it activates, each with distinctive affinities, and thus a particular odorant maps to a particular point in the odor space and is characterized by the corresponding response vector. Recently, odorant response spectra of individual OR molecules have begun to be characterized (Krautwurst et al., 1998; Zhao et al., 1998; Malnic et al., 1999; Speca et al., 1999; Touhara et al., 1999; Wetzel et al., 1999; Araneda et al., 2000), mostly in in vitro systems, but no synopsis of the receptor repertoire for a particular odorant has been possible yet.

Odorant-induced activity of ORs is conveyed to the olfactory bulb, where the primary afferents of olfactory receptor neurons (ORNs) form synapses with projection neurons and local interneurons within the olfactory glomeruli (Shepherd, 1993; Mori et al., 1999). Recent molecular work provides strong evidence that any particular ORN expresses only a single type of OR (Wang et

\footnotetext{
Received June 11, 2001; revised July 19, 2001; accepted July 20, 2001.

This work was supported by a grant from Deutsche Forschungsgemeinschaft, Schwerpunkt Programm Molekulare Sinnesphysiologie to S.I.K.

Correspondence should be addressed to Sigrun I. Korsching, Universität zu Köln, Institut für Genetik, Zülpicher Strasse 47, 50674 Köln, Germany. E-mail: Sigrun.Korsching@uni-koeln.de.

Copyright (ㄷ) 2001 Society for Neuroscience $\quad 0270-6474 / 01 / 218396-12 \$ 15.00 / 0$
}

al., 1998; Malnic et al., 1999) (but see Rawson et al., 2000; Vosshall et al., 2000). Neurons expressing the same OR molecule are scattered within the olfactory epithelium but converge onto single glomeruli as they project to the olfactory bulb (Mombaerts et al., 1996). Thus, a glomerulus integrates odor responses for an individual OR. As a consequence, imaging the odorant-induced activity in the presynaptic (input) compartment of glomeruli at once visualizes the receptor repertoire activated by that particular odorant. Previous work from our lab has demonstrated the feasibility of selectively imaging odorant-induced neuronal activity in the input compartment of glomeruli (Friedrich and Korsching, 1997, 1998), using zebrafish as experimental system. Proteinogenic amino acids (AAs) were found to activate many glomeruli with complex response characteristics (Friedrich and Korsching, 1997).

In this study, we used optical imaging of odorant-induced calcium changes in receptor neuron terminals in the zebrafish olfactory bulb to systematically analyze responses to variations in the chemical structure of AA odorants. Stimuli could be applied repetitively and therefore be compared with high accuracy within a single preparation. AAs are well known behaviorally relevant stimuli for fish, and many structurally related compounds are available. Thus, data obtained at the bulbar level reveal aspects of the structural determinants of AA odorants, as well as the tuning specificities of the AA-responsive glomeruli and their underlying ORs.

\section{MATERIALS AND METHODS}

Adult male zebrafish, Danio rerio ( $>6$ months of age), of the Tü/Tü and $\mathrm{AB} / \mathrm{Tü}$ wild-type strains from our local rearing facility were used for all experiments. Animals were maintained in flow-through tanks, kept under 


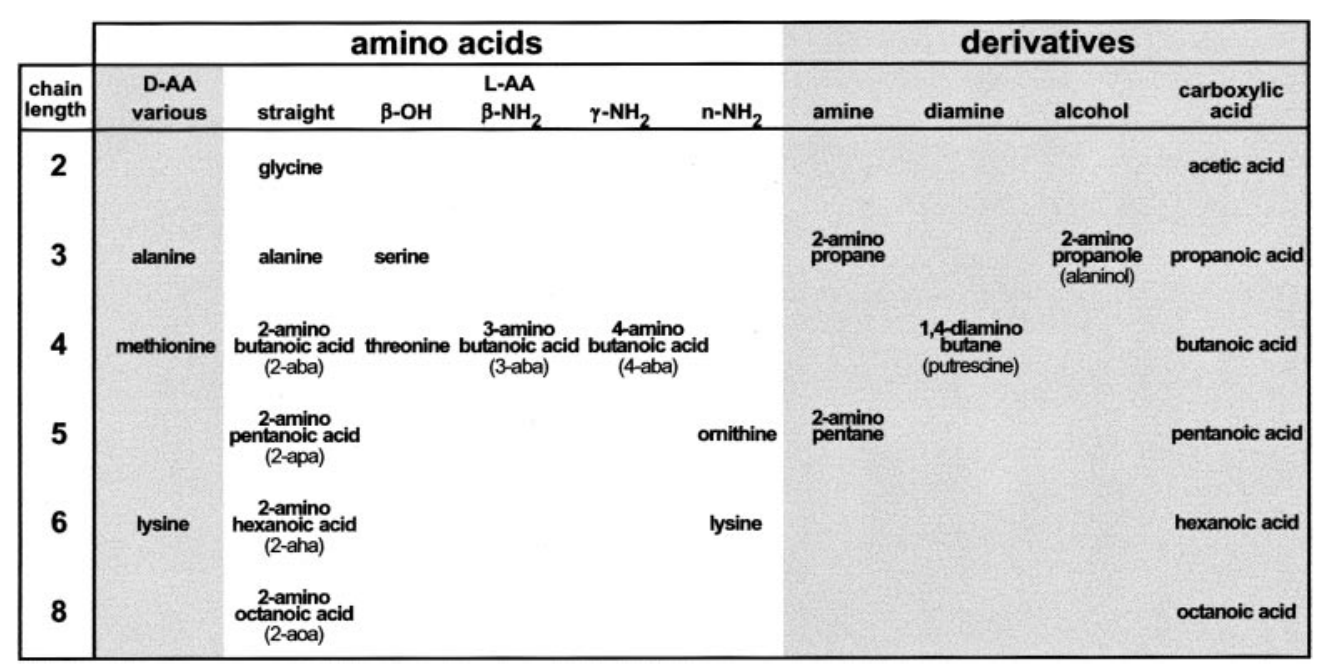

Figure 1. Overview of compounds investigated. The list of all AAs and their analogs tested is arranged according to chain length and functional group. All abbreviations and trivial names used elsewhere are given here. Chain length is given in the first column and refers to the number of carbon atoms in the backbone. Column 2 contains AAs with D-conformation; columns 3-7 contain AAs with L-conformation; columns $8-11$ contain various AA derivatives. a 14/10 hr light/dark cycle and fed on fish flakes (Sera, Heinsberg, Germany).

Dye labeling of primary sensory afferents. Axonal tracing of Calcium Green-1 dextran was performed essentially as described by Friedrich and Korsching (1997). Briefly, sensory afferents were labeled with a $12 \%$ solution of the calcium-sensitive probe Calcium Green-1 dextran [10,000 molecular weight; Molecular Probes, Leiden, The Netherlands; in 0.1\% Triton X-100 (Sigma, Deisenhofen, Germany), 2 mm NaCl). Fish were immobilized with tricaine (Sigma) and wrapped in a wet paper towel, and a volume of $1 \mu \mathrm{l}$ was injected into the fish's left naris. Olfactory cilia recover completely within $2 \mathrm{~d}$ from Triton X-100 treatment, and bulbar fluorescence of ORN terminals is clearly detectable at the same time.

Preparation of explants. A reduced preparation, comprising the olfactory system and the rostral half of the telencephalon, was cut and transferred ventral side down to a coverslip that lined the bottom of a custom-built recording chamber. The preparation was superfused continuously with artificial CSF (ACSF) composed of (in mM): $131 \mathrm{NaCl}, 20$ $\mathrm{NaHCO}_{3}, 2 \mathrm{KCl}, 1.25 \mathrm{KH}_{2} \mathrm{PO}_{4}, 2 \mathrm{MgSO}_{4}, 2.5 \mathrm{CaCl}_{2}, \mathrm{pH} 7.4$, bubbled with $95 \% \mathrm{O}_{2} / 5 \% \mathrm{CO}_{2}$ (Mathieson and Maler, 1988).

Optical imaging of bulbar responses. Preparations were viewed with an inverted microscope (Zeiss, Göttingen, Germany) at 10 or $20 \times$ magnification. Calcium Green-1 fluorescence was detectable at $470 \mathrm{~nm}$ excitation wavelength using a monochromator system (Polychrome II; T.I.L.L. Photonics, Martinsried, Germany). Exposure times varied between 80 and $200 \mathrm{msec}$. Image series of the anterolateral olfactory bulb were acquired at $2 \mathrm{~Hz}$ frame rate before and during stimulation with odorants using a 12 bit cooled CCD camera system $(640 \times 480$ pixel, $2 \times 2$ binning; T.I.L.L. Imago; T.I.L.L. Photonics). Series were fed into a conventional computer and corrected for background and bleaching, and $\Delta F / F$ was calculated using a five-frame prestimulus average as $F$. For illustration purposes, 10 frames comprising the response peak were averaged, lowpass filtered with a $3 \times 3$ Gaussian filter kernel, and false color coded for signal intensity.

Stimulation with odorants. Working concentrations of odorants (see Fig. 1) (Fluka, Steinheim, Germany) were prepared freshly from $10^{-2} \mathrm{M}$ stock solutions each time. Stocks were renewed at least every month and kept frozen at $-20^{\circ} \mathrm{C}$ until use. Alaninol, putrescine, 2-amino propane, and 2-amino pentane were not stored as stock solutions but were prepared freshly each time to avoid any degradation. For stimulation, a volume of $200 \mu \mathrm{l}$ odorant solution was intercalated (HPLC valve; Knauer, Berlin, Germany) into a constant carrier stream of ACSF $(1.5-2.5 \mathrm{ml} / \mathrm{min}$; stable for a given experiment). Odorants reached a peak of $\sim 70 \%$ of the injected concentration at the explant site, as estimated by application of a fluorescent stimulus. The concentrations that are given always refer to the injected odorant concentration. The concentrations that were used varied between $10^{-2}$ and $10^{-7} \mathrm{M}$, the former approaching the solubility limit for 2-amino octanoic acid. Within an experiment, the order of stimulus application was randomized or with increasing concentration (some dose-response curves). It was found that results do not depend on the order of application.

For adaptation experiments the carrier solution was switched to ACSF containing the adapting stimulus at the same concentration as the test stimuli $(100 \mu \mathrm{M})$ and was applied for at least $10 \mathrm{~min}$ before stimulation with another odorant. Readaptation was achieved by switching back to pure ACSF $10 \mathrm{~min}$ before stimuli were retested. During adaptation, images were taken at $30 \mathrm{sec}$ intervals to trace the signal time course.

Image transformations. Activity was nearly always contained within a $200 \times 100$ pixel subframe, which was chosen for further analysis. To obtain a quantitative measure of overall responses, we calculated the spatial extent of the response area. Fluorescence intensity values were thresholded at $\Delta F / F=1 \%$ (corresponding to 2-3 SD of noise level), and the number of all pixels above threshold was determined. This number was normalized to the corresponding value obtained for a reference response within the same experiment. The normalized response areas (nRAs) can then be compared across animals, allowing the results to be averaged over different preparations.

To visualize directly the pattern differences between an image pair, four independent trials recorded in the same preparation were averaged for each stimulus, and the intensity values for each corresponding pair of pixels were subtracted from each other. Negative values were set to 0 (clipped difference). In this way a positive difference image was obtained. Therefore subtraction was always performed in both possible directions.

To obtain a quantitative measure of pattern dissimilarity, we calculated the dissimilarity index $(D I)$ for an image pair (cf. Johnson et al., 1998). DIs were always calculated for image pairs within a preparation but could then be compared across preparations. We first normalized images by a $z$-score transformation and afterward subtracted intensity values pixel-wise from each other. The absolute values of all pixels within these difference images were averaged and yielded a dissimilarity index for each image pair. Lower values represent more similar response patterns, and higher values represent more dissimilar response patterns ( $D I=0$ for identical pictures and $D I \approx 1$ for pure noise pictures). The $D I$ s obtained for repeated trials with the same stimulus $\left(D I_{\text {intertrial }}\right.$, a measure of the noise level) were subtracted from the $D I$ s obtained for trials with pairs of stimuli to obtain a measure of the "specific" pattern dissimilarity $(s D I)$.

\section{RESULTS}

\section{Basic properties of amino acid-induced signals}

We systematically altered chemical features of simple AA stimuli to decipher the structural determinants of the AA-OR interaction. AA consist of an $\alpha$-carbon atom surrounded asymmetrically by an $\alpha$-amino group, an $\alpha$-carboxyl group, an $\alpha$-hydrogen, and a variable residue, which is characteristic for a given AA. With the exception of the $\alpha$-hydrogen, we introduced changes into any of the remaining three moieties or the stereo conformation of the whole molecule (Fig. 1). All structural variations had clear influences on either overall signal intensity or the spatial distribution of signals within the olfactory bulb. Moreover, both parameters were sensitive to changes in stimulus concentration.

Stimulation of the nasal epithelium with any L- $\alpha$-AA (see Fig. 1) evoked broad, multifocal patterns of activity restricted to 


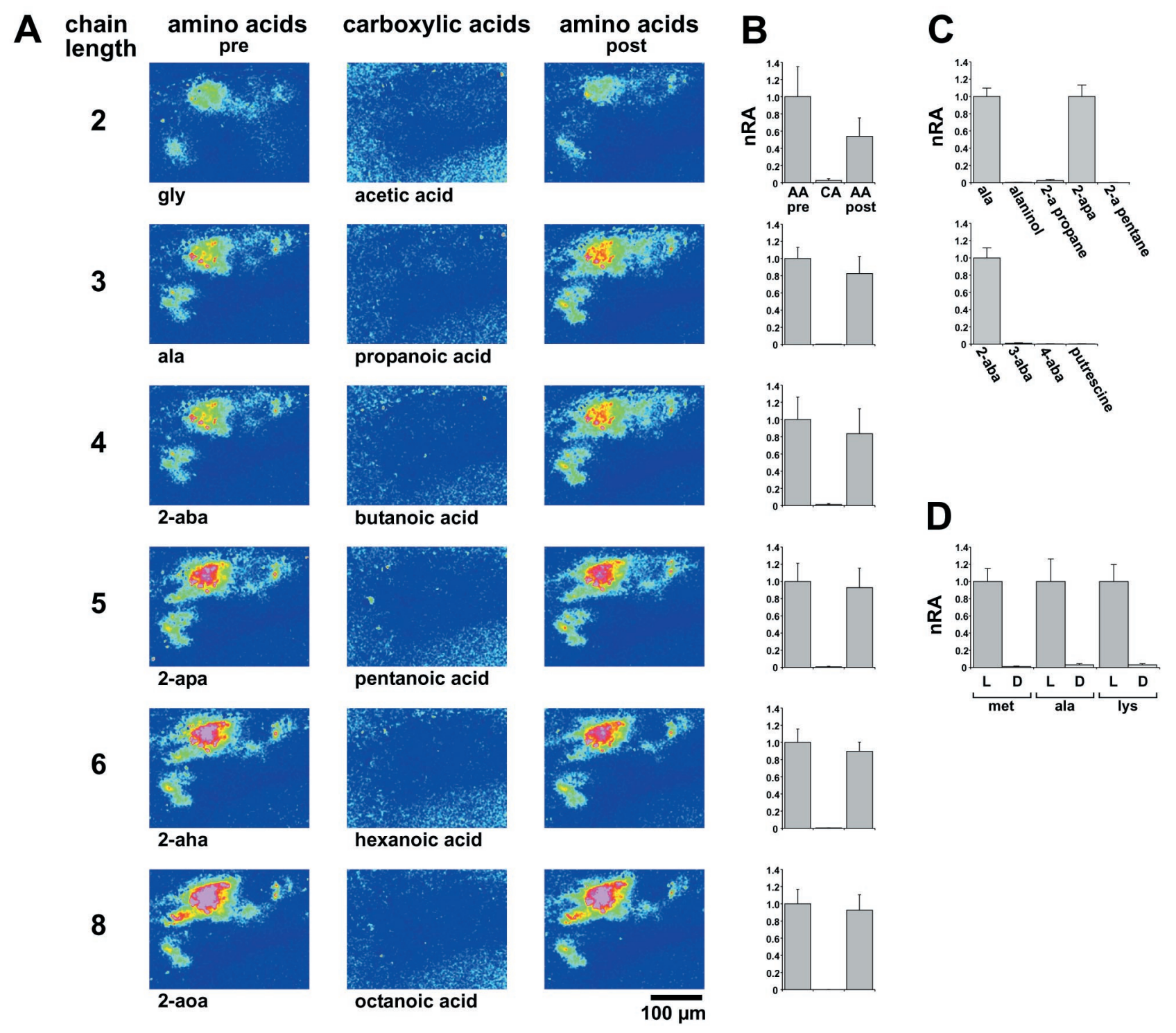

Figure 2. $\mathrm{L}$ - $\alpha$-AA-configuration is required for response. A, Carboxylic acids are compared with AAs in a representative preparation. The anterolateral region of the olfactory bulb is shown. $\Delta F / F$ values are false color-coded (blue to red: -0.5 to $4 \%$ change). Note the lack of response to carboxylic acids. $B$, Quantitation of response areas for carboxylic acids and AAs. Stimulus concentrations were $10^{-4} \mathrm{M}$ for carboxylic acids and AAs. Response areas above threshold $(\Delta F / F>1 \%)$ are normalized $(n R A)$ to the first AA stimulus $(A A$ pre). $C A$, Carboxylic acid (chain length as in $A$ ); $A A$ post, AA stimulus given after stimulation with carboxylic acid. Values shown are means and SEM of four trials (total of 3 preparations). $C$, Quantitation of response areas for other AA analogs. None of the analogs (for abbreviations see Fig. 1) elicited a response, in contrast to the corresponding AAs with the same chain length (used as reference value). Values shown are means and SEM of 3-22 trials (2-5 preparations). For 2-amino pentane and putrescine, two trials were measured. $D$, Quantitation of response areas for L- and D-AAs. Response to the L-conformation is taken as reference value. Note the nearly complete absence of response to the D-conformation. Values shown are means and SEM of eight trials (total of 4 preparations).

the anterolateral subregion of the ventral olfactory bulb. These patterns were highly reproducible upon repetitive stimulation of the same preparation but varied somewhat among different preparations, for either technical or biological reasons (compare Figs. 2, 3, 8, and 9, all of which were recorded from different animals).

\section{Both the $\alpha$-amino group and the $\alpha$-carboxyl group as well as L-conformation are required}

First we wanted to know which moieties of AA are essential for activation of any AA-responsive glomerulus. The $\alpha$-amino group was removed, resulting in carboxylic acids. In many air-breathing vertebrates, homologous series of carboxylic acids have been shown to be potent odorants that could be discriminated easily by the olfactory system (Mori et al., 1992; Laska and Teubner, 1998; Johnson et al., 1999; Rubin and Katz, 1999). A series of carboxylic acids with carbon chain lengths ranging from two (acetic acid) to eight (octanoic acid) were compared with their AA analogs. All carboxylic acids failed completely to evoke signals within the AA-responsive subregion of the olfactory bulb (Fig. $2 A$ ) for all concentrations tested, i.e., between $10^{-5}$ and $10^{-2} \mathrm{M}$. A quantitative evaluation of the response area confirmed this result (Fig. $2 B)$. In contrast, all aliphatic $\alpha$-AAs with corresponding backbone length (glycine to 2-aoa) were potent activators of glomerular responses (Fig. $2 A, B$ ), with thresholds ranging between $10^{-6}$ and $10^{-4} \mathrm{M}$ (see below). The amino group indeed is required specifically in the $\alpha$-position, because $\beta$ - and $\gamma$-positions (3-aba 
and 4-aba, the neurotransmitter GABA), were not stimulatory, in contrast to the corresponding $\alpha$-AA, 2-aba (Fig. 2C).

Changing the carboxyl group to a completely saturated methyl group leads to 2-amino alkanes. 2-Amino propane and 2-amino pentane, the saturated analogs of the AA alanine and 2-apa, did not evoke any response of the AA receptors even at $10^{-2} \mathrm{M}$ (Fig. $2 C$ ). Even the less drastic substitution of the charged carboxyl moiety with a polar hydroxyl group (alaninol) was not able to rescue AA responses (Fig. 2C). A naturally occurring diamine, putrescine (1,4-diamino butane), is set free during rotting processes and therefore might be behaviorally relevant for fish. However, putrescine was not stimulatory (Fig. 2C), in contrast to its strong odor for air-breathing vertebrates.

Because both residues at the $\alpha$-carbon atom, the $\alpha$-amino group and the carboxyl group, are required, we tested whether its stereo conformation is also mandatory. We tested Denantiomeres of three stimulatory AAs, which belonged to different subclasses (basic: lysine; short-chain neutral: alanine; long-chain neutral: methionine). At a concentration of $10^{-4} \mathrm{M}$, all L-isomeres elicited strong responses. The corresponding D-isomeres did not evoke any responses within the ventrolateral olfactory bulb at the same concentration (Fig. 2D). Indeed, up to $10^{-2} \mathrm{M}$, the highest concentration tested, no response was observed. Thus, zebrafish AA receptors do require the Lconfiguration of AA for activation. Inactive compounds do not evoke activity within other bulbar subregions.

As described above, $\alpha$-AA-related compounds do not elicit signals within the AA-responsive subregion of the bulb. Still, these compounds might interact with a different set of ORs and therefore evoke responses within different bulbar subregions. Thus, signals were recorded from the whole olfactory bulb. However, none of the $\alpha$-AA-related compounds tested (carboxylic acids, amines, putrescine, alaninol, 3-aba, and 4-aba) and none of the D-isomeres (D-lys, D-ala, and D-met) elicited a signal in any subregion of the olfactory bulb (data not shown). AA signals arise in the upper third along the ventrodorsal axis of the bulb; nevertheless, they were clearly visible in all focal planes extending through the entire dorsoventral axis of the olfactory bulb, despite some blurring of the signal in the dorsalmost optical sections (tested with alanine at $10^{-3}$ and $10^{-4} \mathrm{M}$ ). In contrast, for the closely related alaninol, no signals were observed in any of the corresponding focal planes, regardless of the concentration tested $\left(10^{-2}-10^{-5} \mathrm{M}\right.$; data not shown).

\section{Inactive compounds may influence signals elicited by the related active compounds}

L-AA responses could be obtained repetitively before and after application of D-isomeres (data not shown). AAs were effective before and after stimulation with carboxylic acids (Fig. 2A,B) or amines (data not shown), and their responses did not differ noticeably in either strength or spatial distribution of the signals, indicating that the inactive compounds did not elicit any toxic effects and that the absence of signals is indeed caused by a failure to activate the AA receptors. However, simultaneous application of active and inactive compounds altered the signal in some cases. For example, concomitant application of alaninol and alanine reduced the response area to $50 \%$ ( $p<0.005 ; n=9$ trials; Student's $t$ test) compared with alanine applied alone. Simultaneous application of propanoic acid and alanine increased the response area to $154 \%$ ( $p<0.005 ; n=7$ trials). Thus, propanoic acid seems to elicit a facilitatory effect, whereas alaninol acts as antagonist.
Taken together, our data have shown that all structural motives of the AA backbone region ( $\alpha$-amino as well as the $\alpha$-carboxyl group and the L-conformation) are absolutely required for the activation of AA-responsive ORs in zebrafish. All of the tested analogs that differed in any of these aspects failed completely to elicit a specific response.

\section{Increases in carbon chain length successively recruit active glomeruli}

Modifications of the AA side chain, on the other hand, resulted in changed activity patterns rather than a complete loss of activity. To compare the effects of small structural changes, we first analyzed the effects of increasing carbon chain length with a homologous series of unbranched aliphatic L-AAs ranging from glycine (chain length of two) to 2-aoa (chain length of eight). Representative experiments are shown in Figures $2 A$ and $3 A$.

Even the smallest AA, glycine, activated $\sim 25$ different glomeruli in the olfactory bulb (counted in seven animals). As carbon chain length increases, new foci of activity are successively recruited. Glomeruli activated by shorter AAs were activated to a higher extent by longer compounds. This unexpected result is a general feature, although in some experiments a few glomeruli preferring shorter AAs have also been detected (see Fig. $6 D$ for an example). Thus, signal complexity and overall signal intensity generally increase with increasing backbone length. A quantitative evaluation for all experiments shows a continuous increase of response area with chain length (see Fig. 4). We stress that the response is not limited to the proteinogenic AAs glycine and alanine; indeed, even stronger responses are observed for the nonproteinogenic AAs (Figs. 2A, 3).

For a visualization of the tuning characteristics of individual glomeruli, we performed pixel-wise subtraction of signal intensities between pairs of stimuli as described in Materials and Methods. In such subtraction pictures, foci are visible when the underlying glomerulus responds differentially to the two stimuli. Difference foci were visible only where responses obtained with shorter AAs were subtracted from those elicited with longer compounds (Fig. 3B) but not in the inverse subtraction, confirming the qualitative observation. All one-carbon extensions in chain length, except that between ala and 2-aba, elicit activity in one to several additional foci. Some foci reflect sharp transitions, e.g., a group of glomeruli not visible in the 2-aba response, but prominent in the 2-apa response (Fig. 3B). Even the longest AA, 2-aoa, activates an additional glomerulus, despite its generally somewhat weaker response. It should be noted that the exact number and position of such foci are not constant between animals, for technical and/or biological reasons. However, foci with similar response characteristics and positions were observed repeatedly in different animals.

Thus some foci, and by inference their underlying receptors, appear sharply tuned to particular minimal chain lengths, whereas others exhibit broader tuning curves visualized as gradual increases with increasing chain length. At least five different foci orgroups of foci are involved in the response to neutral aliphatic AAs, distinguishable by their onset of response at different chain lengths. For a listing of response categories that occur, see Table 1.

\section{The discriminating ability for chain length depends on concentration}

To enable comparisons of pattern differences across animals, we calculated pattern dissimilarity between different chain lengths as difference index DI and averaged DI across all preparations. The 


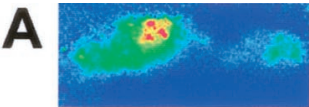

gly

B
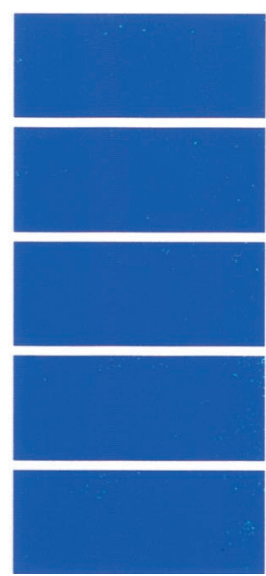

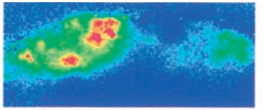

ala
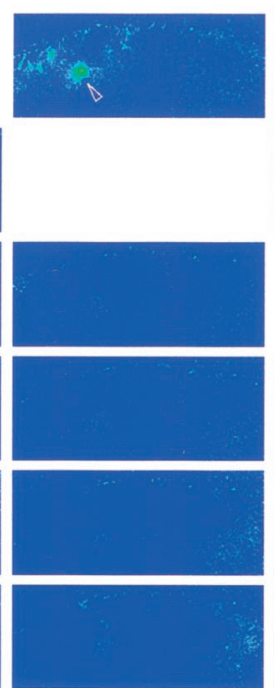

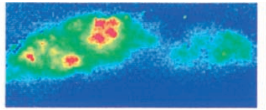

2-aba
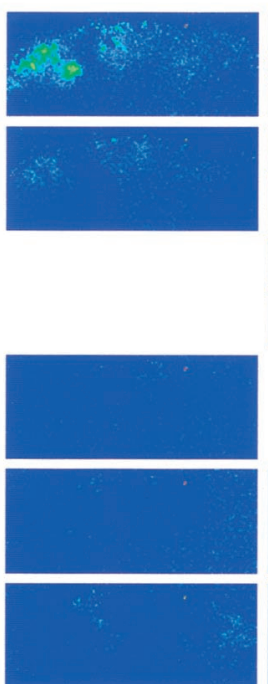

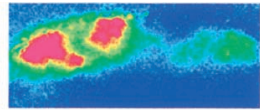

2-apa
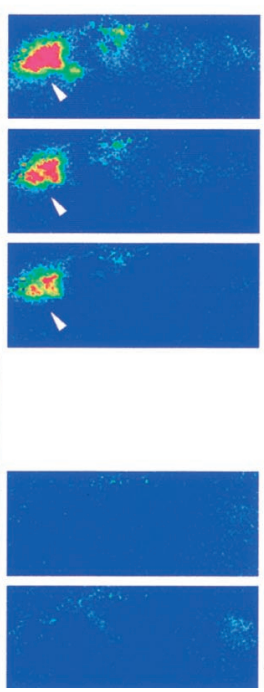

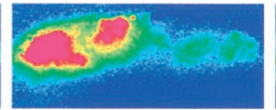

2-aha
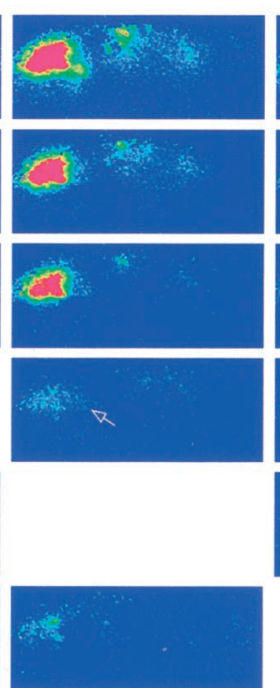

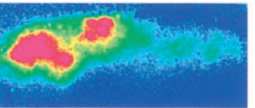

2-aoa

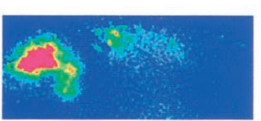

- gly

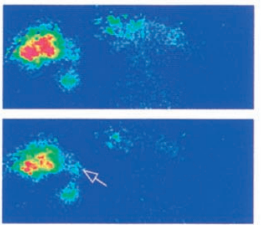

- ala

- 2-aba
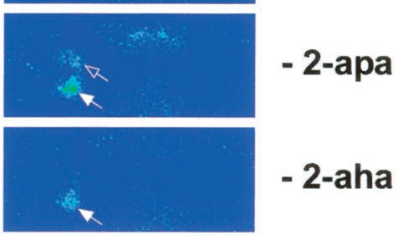

- 2-aha

$100 \mu \mathrm{m}$

Figure 3. Increases in carbon chain length successively recruit active glomeruli. $A$, Images are averages of four trials. Shown is $\Delta F / F$, color-coded as before (color scale -0.5 to $3 \%$ ). Increasing chain length recruits additional glomeruli and increases signal intensity in already activated glomeruli. $B$, Difference images for the data shown in $A$ (color scale $0-2.5 \%$ ). All possible difference images are arranged as matrix. In the top triangle, the shorter AA was subtracted; in the bottom triangle, the longer one was subtracted. Difference foci are visible only in the top triangle. The weak signal in the 2-aoa minus 2-aha subtraction may reflect a reduced actual concentration of 2-aoa attributable to solubility problems. The ala response already shows a clear difference focus (open arrowhead). The addition of a single carbon atom at the transition between 2-aba and 2-apa gives rise to several additional activity foci (arrowhead). These foci lie close together and are seen in any difference pattern, where 2-aba or shorter stimuli were subtracted. An additional focus (open arrow) begins to be visible at the 2-apa to 2-aha transition (somewhat obscured by noise in the adjacent area) and becomes prominent in the 2-aoa versus 2-apa comparison. A medium-sized focus (arrow) is activated uniquely by 2-aoa, because it is not observed with any of the shorter AAs tested, including 2-aha. Scale bar, $100 \mu \mathrm{m}$.

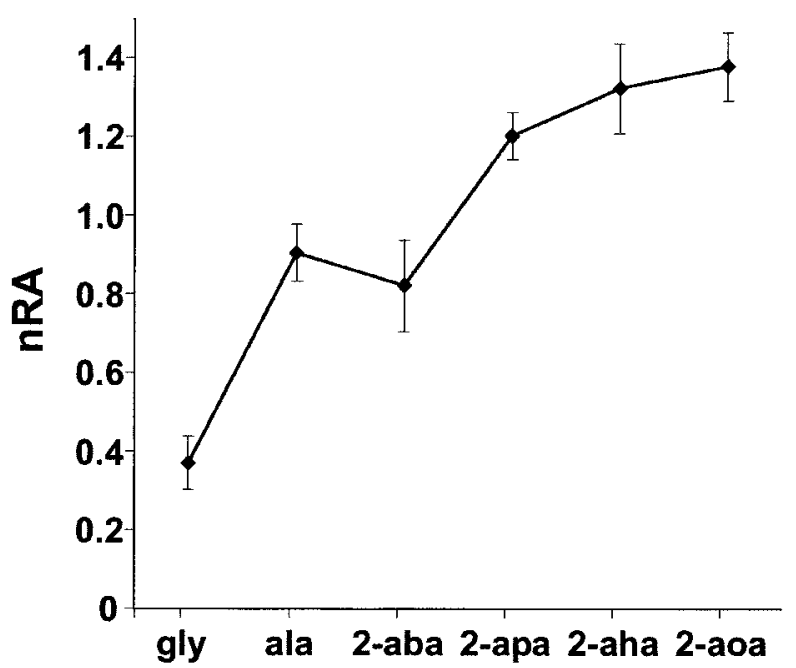

Figure 4. Response area as function of chain length. Threshold was set at $\Delta F / F=1 \%$; area above threshold was quantified and normalized to the average area of all chain lengths. Note the steady increase of response area with increasing chain length. The slight drop between alanine and 2 -aba is not significant. Values shown are means \pm SEM of 16 trials (total of 9 preparations).

contribution of intertrial variability was evaluated by calculating the dissimilarity indices for repeated trials of the same stimulus for all AAs and all concentrations. These values represent the noise level in the dissimilarity analysis.

The discriminating ability for chain length was pronounced at the intermediate concentrations $10^{-4}$ and $10^{-5} \mathrm{M}$, with significance values ranging between $p<0.05$ and $p<0.0001$ (Fig. 5). At both higher and lower concentrations, chain length is not discriminated significantly, albeit for different reasons. At lower concentrations, signals approach noise levels (Fig. 6). At higher concentrations, signal-to-noise ratio is high, but response patterns for different chain lengths become more similar, possibly because saturation range is reached for an increasing number of AA receptors underlying these response patterns (compare Fig. 6), making them less distinctive.

Response patterns at the "discriminating" concentrations $10^{-4}$ and $10^{-5} \mathrm{M}$ tend to show increasing dissimilarity for increasing differences in carbon chain length (Fig. 5). Thus, larger differences in chain length are better resolved. However, even a change of one methylene group, a small difference in stimulus structure, can elicit a significantly different response pattern (Fig. 7).

\section{Differences in concentration do not compensate for differences in chain length}

It is conceivable that pattern dissimilarity for chain-length differences might be compensated for by differences in concentration; e.g., glycine at higher concentrations might elicit a pattern undistinguishable from 2-aba at lower concentrations.

Therefore we examined the concentration dependence of the response patterns for all six AAs. Typical results for two very similar AAs and a more distant one (gly, ala, and 2-aha, respectively) are shown in Figure 6. For all odorants, signal complexity grew with increasing concentration. At the lowest concentration tested $\left(10^{-7} \mathrm{M}\right)$, none of the stimuli was strong enough to elicit glomerular responses. With increasing concentrations, the longer 


\begin{tabular}{|c|c|c|c|c|c|c|}
\hline \multirow[b]{3}{*}{ Category } & \multicolumn{6}{|c|}{ Chain length } \\
\hline & 2 & 3 & 4 & 5 & 6 & 8 \\
\hline & \multicolumn{6}{|c|}{ Response properties of glomeruli } \\
\hline 1 & $\mathrm{r}$ & $\mathrm{t}$ & $\mathrm{t}$ & $\mathrm{t}$ & $\mathrm{t}$ & $\mathrm{t}$ \\
\hline 2 & $\mathrm{n}$ & $\mathrm{r}$ & $\mathrm{t}$ & $\mathrm{t}$ & $\mathrm{t}$ & $\mathrm{t}$ \\
\hline 3 & $\mathrm{n}$ & $\mathrm{n}$ & $\mathrm{n}$ & $\mathrm{r}$ & $\mathrm{t}$ & $\mathrm{t}$ \\
\hline 4 & $\mathrm{n}$ & $\mathrm{n}$ & $\mathrm{n}$ & $\mathrm{n}$ & $\mathrm{r}$ & $\mathrm{t}$ \\
\hline \multirow[t]{2}{*}{5} & $\mathrm{n}$ & $\mathrm{n}$ & $\mathrm{n}$ & $\mathrm{n}$ & $\mathrm{n}$ & $\mathrm{r}$ \\
\hline & $\mathrm{nNH}_{2}$ & & & & & \\
\hline 6 & $\mathrm{t}$ & $\mathrm{t}$ & & & & \\
\hline 7 & $\mathrm{t}$ & $\mathrm{n}$ & & & & \\
\hline 8 & $\mathrm{r}$ & $\mathrm{n}$ & & & & \\
\hline 9 & $\mathrm{n}$ & $\mathrm{t}$ & & & & \\
\hline 10 & $\mathrm{n}$ & $\mathrm{n}$ & & & & \\
\hline
\end{tabular}

Categories of AA-responsive glomeruli based on differential tuning properties of individual glomeruli. Top: $r$, required minimal chain length; $t$, tolerated (all other chain lengths leading to activation); n, no response (compound is not sufficient for activation). Five different tuning properties were found in this analysis. Bottom: influence of functional groups in the AA side chain; $r$, required (presence of functional group is required); $t$, tolerated (response in presence and absence of functional group); $\mathrm{n}$, not tolerated (functional group needs to be absent for response). Note that not all possible combinations exist. Five different tuning categories were found for functional groups. At least some are further subdivided because of different dependence on chain length (see Fig. 8). The maximal possible number of $5 \times 5$ categories is not reached, however, because not all combinations of chain-length preference and functional-group preference are realized (e.g., category 4 may be subdivided by categories 7,8 , and 10 , but is mutually exclusive with categories 6 and 9). The total number of categories represents a minimal estimate for the total number of different receptors.

chain-length stimuli were the first that reached threshold. Successively shorter stimuli reached threshold with increasing concentrations. At $10^{-2} \mathrm{M}$, the highest concentration tested, signals became more similar to each other.

For quantitative analysis, we first established dose-response curves of many individual activity foci for all six AA stimuli (Fig. 6 and data not shown). Results for three different AAs, gly, ala, and 2-aha, for each of four foci, are illustrated (Fig. 6). Most of these dose-response curves are sigmoidal, with their rise phase spanning two to three logarithmic units, in good accordance with the expectation for receptor ligand interaction (Sveinsson and Hara, 1990). These curve shapes are consistent with the notion that individual foci represent the contribution of a single type of OR. Some dose-response curves do not saturate, possibly because of insufficient binding affinity or because responses of two different foci could not be resolved optically. Note that individual foci in most cases distinguish clearly between different AAs, with 5- to 50-fold differences in half-maximal activation values (Fig. 6). Different foci need up to 10 -fold differences in concentration to reach half-maximal values for the same AA. As a consequence, we never observed a combination (AA and concentration) with a response identical to that of another combination. In other words, even the limited set of four foci appeared sufficient to distinguish between highly related stimuli.

To quantify overall pattern similarity as a function of concomitant changes in chain length and concentration, we obtained dissimilarity indices for relative differences of chain length (up to two carbon atom difference) and concentration (up to 100-fold), using as reference the 2-aba response at $10^{-4} \mathrm{M}$ concentration (Fig. 7). Note that in this surround matrix for pattern similarity, concentration differences give rise to $s D I$ values in the range of
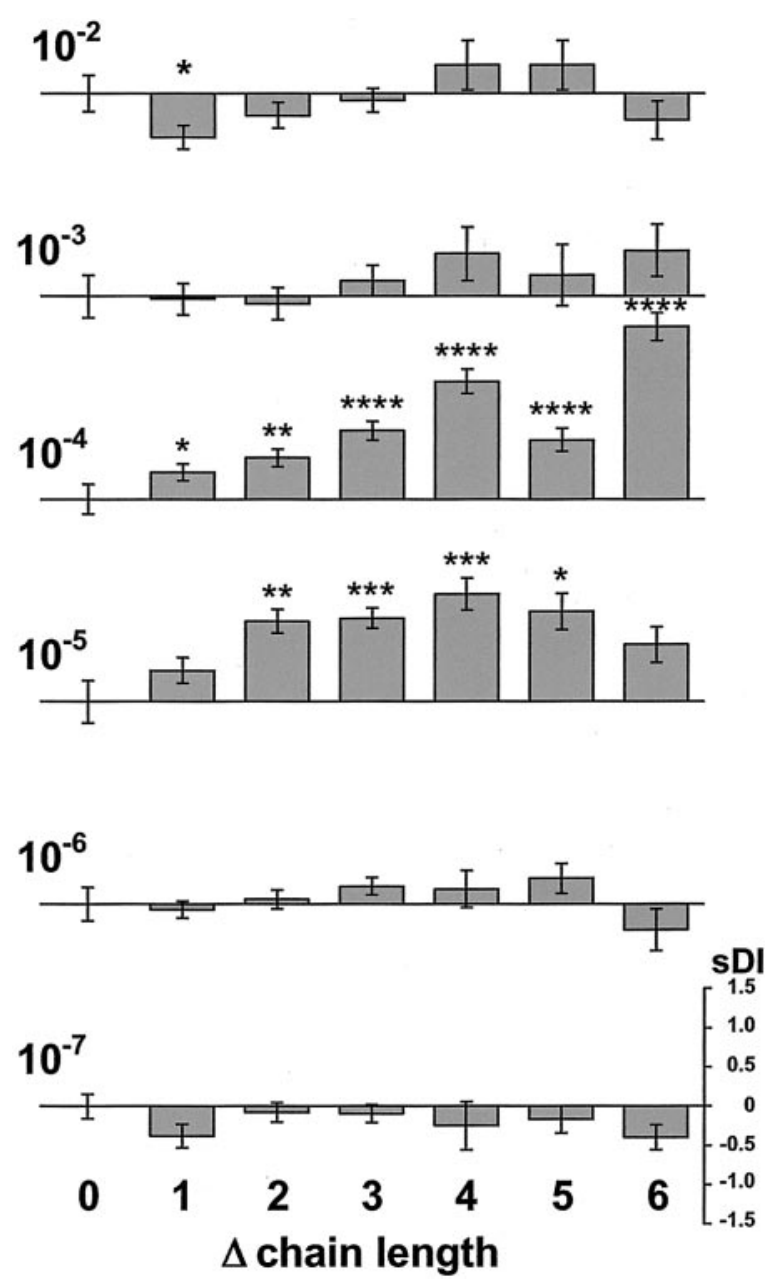

Figure 5. Quantitative analysis of the discriminating ability for neutral AAs at different concentrations. Specific difference indices $\left(s D I=D I_{\text {chain }}\right.$ length pair $\left.-D I_{\text {intertrial }}\right)$ as function of chain length difference $\left(\Delta_{\text {chain length }}=\right.$ $0-6)$ are shown for concentrations between $10^{-7}$ and $10^{-2} \mathrm{M}$. The concentration (molar) is indicated to the left of the corresponding bar graph. A particular chain-length difference represents data from different stimulus pairs, e.g., $\Delta_{\text {chain length }}=4$ contains data from image pairs gly/aha and 2-aba/2-aoa. The sDI scale to the bottom right is valid for all bar graphs. $s D I$ values are given as means \pm SEM. Stimulus concentration drastically affects the discriminating ability for differences in chain length. Discriminating ability is pronounced only at $10^{-4}$ and $10^{-5}$ M. Pattern dissimilarity increases with increasing difference in carbon chain length. Significance levels of $s D I$ values are indicated by asterisks $\left({ }^{*} p<0.05 ; * * \mathrm{p}<\right.$ $0.005 ; * * p<0.001 ; * * * p<0.0001)$.

those caused by differences in chain length. Thus, compensation of one parameter by the other appears conceivable. Nevertheless, all combinations of chain length and concentration differences are significantly more dissimilar than the corresponding noise levels, although some combinations give rise to lower dissimilarity than the respective one-dimensional changes of either chain length or concentration (Fig. 7). Very similar results are obtained using shorter AAs as reference value (Fig. 7, ala; and data not shown). Longer AAs, although generally quite similar, show a tendency to impaired discrimination in comparisons with long AAs (Fig. 7, 2-apa; and data not shown), possibly because with increasing chain length the relative importance of another added methylen group diminishes. Thus, in the vast majority of cases, pattern dissimilarity for chain-length differences is partially reduced but not eliminated by counteracting differences in concentration. 

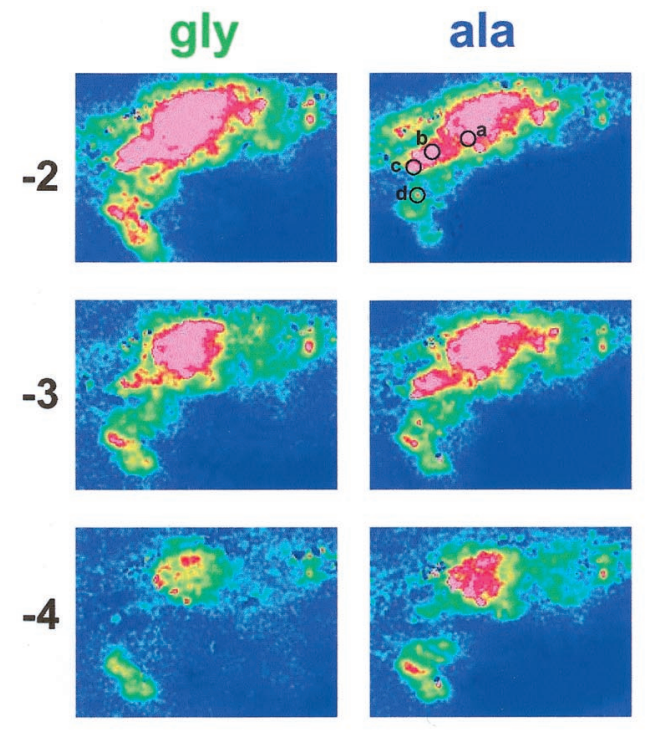

Figure 6. Concentration dependence of responses to three aliphatic AAs. From top to bottom, decreasing stimulus concentration (given as $\log$ conc. $[M]$ ), responses to gly, ala, and 2-aha are shown in false color (color scale -0.5 to $3.5 \%$ ). Dose-response curves for foci $a-d$ (encircled in the image for $10^{-2} \mathrm{M}$ ala) are shown for all three AAs (color coded). Note that focus $c$ does not saturate for any of the three AAs, in contrast to foci $a$, $b$, and $d$. Same preparation as shown in Figure 2.
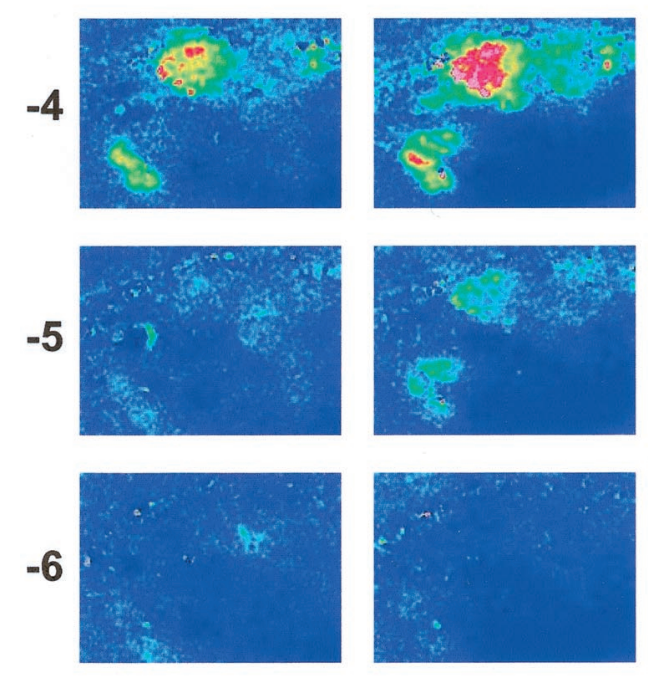
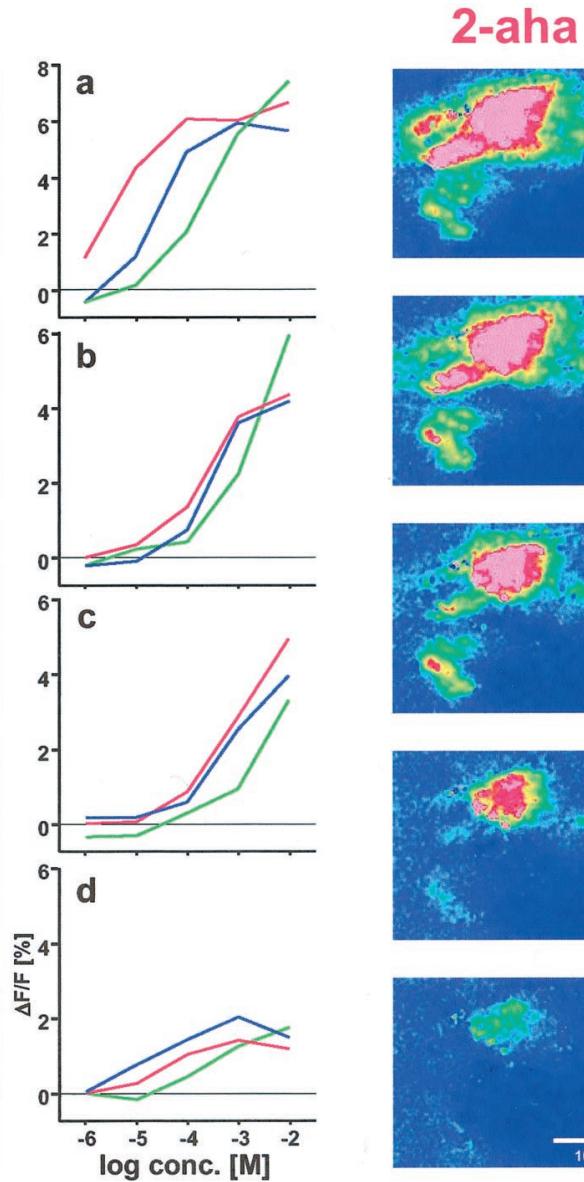
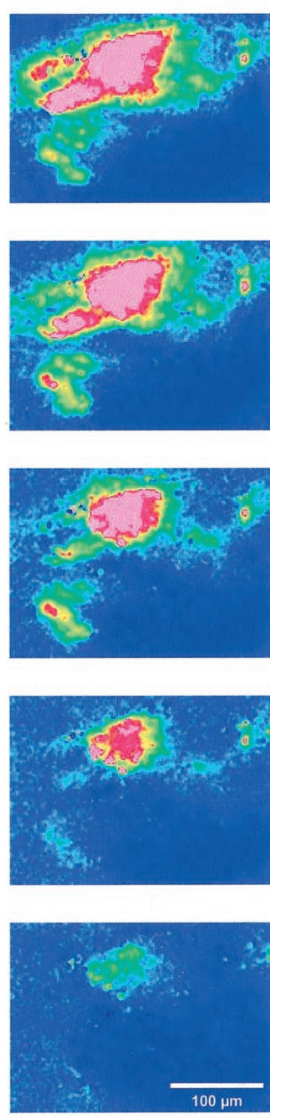

Taken together, our data indicate that at the level investigated, the zebrafish olfactory system has a high discriminatory power, allowing for the distinction of closely related compounds over a wide concentration range. Whether later stages of olfactory signal processing keep all this information remains to be seen. It will be interesting to determine which degree of pattern differences (i.e., $s D I$ level) can manifest itself in behavioral tests of odor discrimination.

\section{Systematic variations of the side chain functional group in amino acids}

We wanted to know how different functional groups, i.e., particular chemical features, are encoded within the activated receptor repertoire. To that end, we compared effects of small variations in the side group at a particular backbone length. This comparison was repeated at another carbon chain length to evaluate whether results might be generalized. The presence and absence of a terminal amino group were examined using the pairs ornithine/ 2-apa and lysine/2-aha (carbon chain lengths 5 and 6 , respectively). The presence and absence of a $\beta$-hydroxyl group were examined with the pairs serine/alanine and threonine/2-aba (carbon chain lengths 3 and 4, respectively).

\section{The presence as well as the absence of a terminal amino group are represented by distinct glomeruli}

Responses elicited by the two basic AAs were located exclusively within the ventrolateral olfactory bulb [for lysine, confirming results obtained by Friedrich and Korsching (1997)]. Response patterns generated by ornithine and lysine appeared similar to each other but were evidently different from responses obtained with the corresponding neutral AAs 2-apa and 2-aha (Fig. 8A). Responses of the neutral AAs extended more medially than the ornithine/lysine signals. Moreover, the ornithine/lysine responses consisted of more distinct foci than the responses to 2-apa and 2-aha. Several foci were activated by all four AAs; i.e., they possess a relatively broad tuning curve.

Subtraction analysis revealed foci specific either for a combination of chemical features or for a particular chemical feature (Figs. $8 A, 9$, Table 1). Some glomeruli require the presence of the terminal amino group independently of the particular chain length examined. These detect single features of the side chain, whereas other glomeruli only recognize the terminal amino group at a particular position, i.e., chain length. These might be labeled "holistic" detectors, because they require both a particular chain length and a particular functional group for activation. To our surprise we also detected glomeruli that require the absence of the $\mathrm{N}$-terminal amino group to be activated (Fig. $8 A$, Table 1 ). Again, some of these generalize for both chain lengths examined, whereas others require a particular chain length. Some glomeruli are activated by both the basic AAs and the corresponding neutral AAs; i.e., they tolerate but do not require the presence of the functional group (Table 1). In all three categories (required, not tolerated, tolerated), differential dependence on chain length is observed, supporting the presence of more than three different ORs underlying the observed responses. Thus many glomeruli with distinct response properties have a share in the representation of a single AA. 

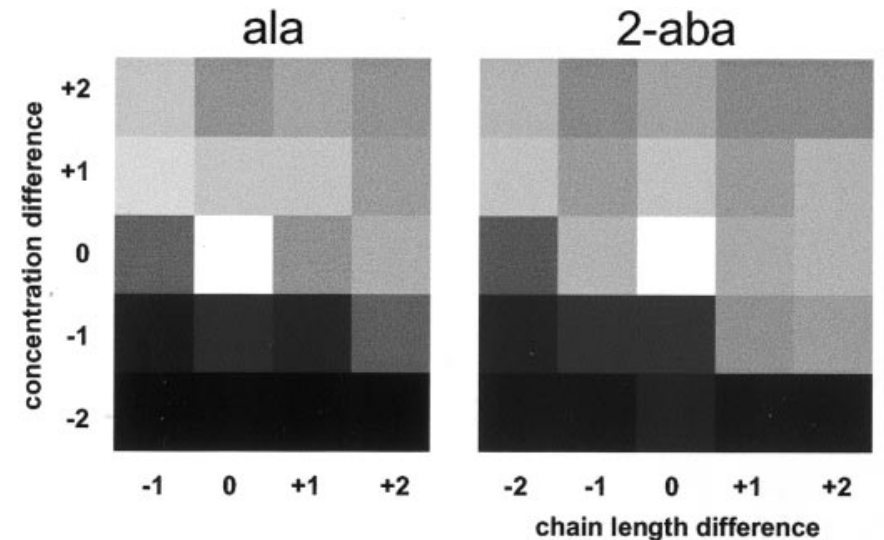

\begin{tabular}{|r|r|r|r|}
\hline $\mathbf{0 . 1 4 1}$ & $\mathbf{0 . 2 1 8}$ & $\mathbf{0 . 1 8 9}$ & $\mathbf{0 . 2 1 6}$ \\
\pm 0.039 & \pm 0.0042 & \pm 0.041 & \pm 0.034 \\
0.0088 & 0.0009 & 0.0017 & 0.0004 \\
\hline $\mathbf{0 . 1 1 2}$ & $\mathbf{0 . 1 4 4}$ & $\mathbf{0 . 1 3 6}$ & $\mathbf{0 . 1 9 7}$ \\
\pm 0.043 & \pm 0.041 & \pm 0.046 & \pm 0.039 \\
0.0291 & 0.0068 & 0.0145 & 0.0035 \\
\hline $\mathbf{0 . 2 9 2}$ & $\mathbf{0 . 0}$ & $\mathbf{0 . 2 1 1}$ & $\mathbf{0 . 1 6 3}$ \\
\pm 0.04 & \pm 0.035 & \pm 0.041 & \pm 0.042 \\
0.0002 & & 0.0023 & 0.006 \\
\hline $\mathbf{0 . 4 7 2}$ & $\mathbf{0 . 3 9 8}$ & $\mathbf{0 . 4 3 8}$ & $\mathbf{0 . 2 9 3}$ \\
\pm 0.019 & \pm 0.039 & \pm 0.036 & \pm 0.04 \\
$<0.0001$ & $<0.0001$ & $<0.0001$ & $<0.0001$ \\
\hline $\mathbf{0 . 5 1 3}$ & $\mathbf{0 . 5 1 9}$ & $\mathbf{0 . 5 2 1}$ & $\mathbf{0 . 5 1 7}$ \\
\pm 0.019 & \pm 0.032 & \pm 0.023 & \pm 0.038 \\
$<0.0001$ & $<0.0001$ & $<0.0001$ & $<0.0001$ \\
\hline
\end{tabular}

\begin{tabular}{|r|r|r|r|r|}
\hline $\mathbf{0 . 1 5 3}$ & $\mathbf{0 . 2 3 2}$ & $\mathbf{0 . 1 8 9}$ & $\mathbf{0 . 2 2 1}$ & $\mathbf{0 . 2 3 0}$ \\
\pm 0.03 & \pm 0.036 & \pm 0.036 & \pm 0.029 & \pm 0.031 \\
0.0184 & 0.0023 & 0.0088 & 0.0017 & 0.0012 \\
\hline $\mathbf{0 . 1 3 6}$ & $\mathbf{0 . 1 9 9}$ & $\mathbf{0 . 1 3 9}$ & $\mathbf{0 . 2 0 1}$ & $\mathbf{0 . 1 6 0}$ \\
\pm 0.008 & \pm 0.036 & \pm 0.036 & \pm 0.033 & \pm 0.019 \\
0.0184 & 0.006 & 0.0260 & 0.004 & 0.006 \\
\hline $\mathbf{0 . 2 9 8}$ & $\mathbf{0 . 1 5 3}$ & $\mathbf{0 . 0}$ & $\mathbf{0 . 1 7 4}$ & $\mathbf{0 . 1 6 0}$ \\
\pm 0.033 & \pm 0.041 & \pm 0.047 & \pm 0.03 & \pm 0.019 \\
0.0003 & 0.0088 & & 0.006 & 0.0046 \\
\hline $\mathbf{0 . 4 2 8}$ & $\mathbf{0 . 3 7 0}$ & $\mathbf{0 . 3 9 2}$ & $\mathbf{0 . 2 2 1}$ & $\mathbf{0 . 1 9 8}$ \\
\pm 0.026 & \pm 0.04 & \pm 0.04 & \pm 0.04 & \pm 0.037 \\
$<0.0001$ & $<0.0001$ & $<0.0001$ & 0.0017 & 0.004 \\
\hline $\mathbf{0 . 4 5 4}$ & $\mathbf{0 . 4 6 2}$ & $\mathbf{0 . 4 4 7}$ & $\mathbf{0 . 4 5 4}$ & $\mathbf{0 . 4 5 1}$ \\
\pm 0.025 & \pm 0.033 & \pm 0.03 & \pm 0.037 & \pm 0.034 \\
$<0.0001$ & $<0.0001$ & $<0.0001$ & $<0.0001$ & $<0.0001$ \\
\hline
\end{tabular}

\section{Only the absence of a $\beta$-hydroxyl group is represented by distinct glomeruli}

Responses elicited by the two polar AAs were located exclusively within the ventrolateral olfactory bulb [for serine, confirming results obtained by Friedrich and Korsching (1997)]. Response patterns generated by the $\beta$-hydroxyl AAs, serine and threonine, were evidently different from signals obtained with alanine and 2-aba and appeared less extended than those of their corresponding aliphatic counterparts (Fig. 8B). Difference analysis clearly showed that the unsubstituted AAs activated more glomeruli, and to a larger extent, than the $\beta$-hydroxyl AAs. Some glomeruli recognized all four components and did not distinguish between the presence and absence of the functional group. However, of those glomeruli that do distinguish between the presence and absence of the $\beta$-hydroxyl group, we only observed glomeruli requiring the absence of the $\beta$-hydroxyl group (independent of or dependent on chain length, respectively), but never detected glomeruli specific for the presence of the $\beta$-hydroxyl group. Thus the receptor repertoire for the polar AAs appears more restricted than that for the corresponding unpolar AAs and may be a subset of the latter repertoire. At least three different ORs seem to be involved in the response to ala. By comparing tuning properties between polar and basic AAs, further subdivisions in the receptor repertoire become visible (Table 1).

\section{Glomeruli with related response properties tend to be clustered}

AA-responsive glomeruli with related tuning curves-similar chain length requirements or similar functional group requirements-tend to be grouped together (compare Figs. 2, 3, 8, and 9). Thus the spatial arrangement of glomerular response patterns is not random but chemotopic, even for small changes in odorant structure. A similar tuning curve might indicate a similar structure, i.e., sequence of the underlying OR. Consistent with this hypothesis, it has been observed in mammals that similar ORs

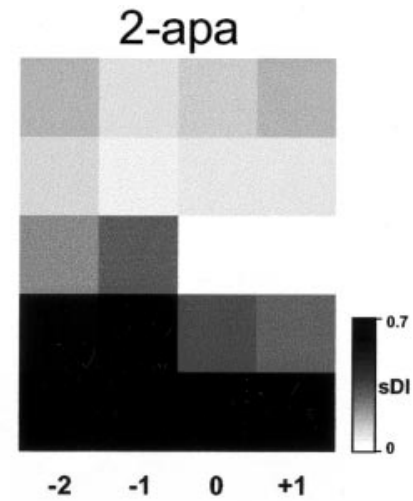

Figure 7. Concentration differences do not compensate for chain-length differences. Pattern dissimilarity is expressed as $s D I$ and given as function of both chain-length difference and concentration difference. Surround matrices $\left(\Delta_{\text {chain length }}=-2\right.$ to +2 and $\Delta_{\text {concentration }}=10^{-2}$ to 100 -fold) are shown in gray scale in the top row. Reference images are from ala (left panel), 2-aba (middle panel), and 2-apa (right panel) at $10^{-4} \mathrm{M}$ concentration. The surround matrix is not complete for ala and 2-apa, e.g., $\Delta_{\text {chain length }}=-2$ is not possible for ala. The bottom row contains for each gray square the numerical value of the $s D I$ (bold) $\pm \mathrm{SEM}$ and the significance level (Mann-Whitney $U$ test; 14 pairings/value, 17 pairings for $D I_{\text {intertrial }} ; p>0.05$ is depicted as not significant; n.s.). Data are from three preparations with six trials total per stimulus. This two-dimensional representation shows that in most cases chain-length differences may not be compensated by differences in concentration, and vice versa. project to neighboring glomeruli (Tsuboi et al., 1999; Strotmann et al., 2000). Accordingly, a certain level of chemotopic representation is observed in mammals as well (cf. Johnson and Leon, 2000; Uchida et al., 2000; Meister and Bonhoeffer, 2001).

\section{Cross-adaptation resembles simple mathematical calculations}

Because odor responses desensitize quickly, cross- adaptation within a group of related odorants can be used to obtain an estimate for the minimal number of different receptors available for neuronal representation of this odorant group. We applied this technique to obtain an independent evaluation of our conclusions drawn from the image subtraction analysis. We adapted the explant by continuously superfusing it with any one of four stimuli (2-apa, 2-aha, ornithine, and lysine) and successively tested all four stimuli again.

Responses to the continuous stimulus resemble those evoked by transient stimulation. Signals rise over the first minute of stimulation and decay within the following $2 \mathrm{~min}$. Within 3-4 min, baseline is restored; i.e., complete adaptation has occurred. Reversal of adaptation is complete after $10 \mathrm{~min}$ without stimulus. Directly after adaptation a stimulus pulse with the adapting odorant did not elicit a specific signal (Fig. 9). Stimulation with other odorants revealed unique activity patterns that depended on the relatedness of the test stimulus to the adapting stimulus (Fig. 9). These response patterns exhibit a striking similarity to the subtraction images described above, the adapting stimulus corresponding to the subtracted image. Figure 9 shows a typical experiment (note the remarkable similarity of the basic, nonadapted patterns to those of Fig. 8, which were obtained with another animal). All categories of tuning properties (Table 1) observed in the subtraction analysis (Fig. 8) could be found in the adapted response patterns. These experiments show first that calcium imaging is an excellent method for quantitative studies of adaptation and cross-adaptation. Second, they provide strong 

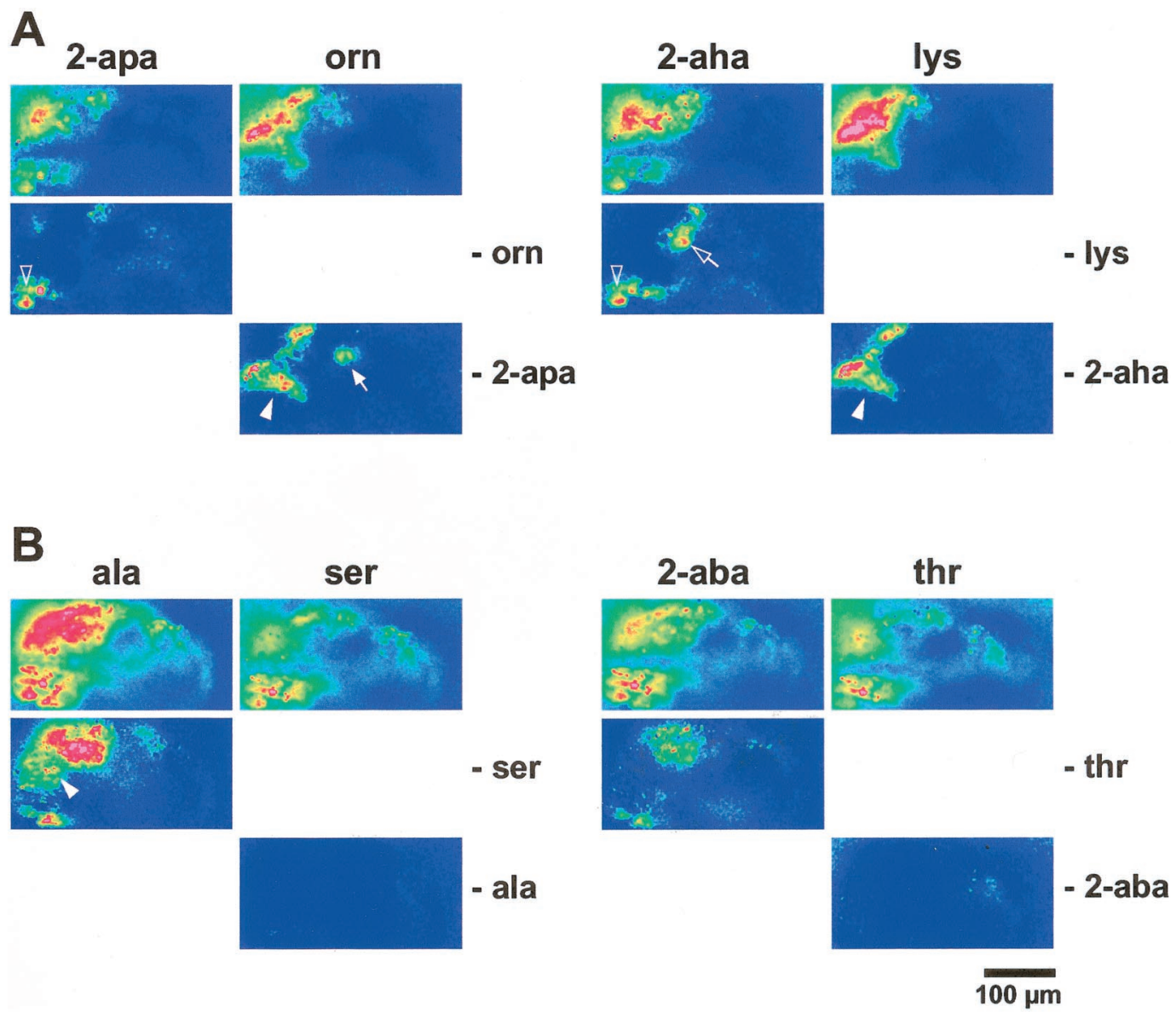

Figure 8. Differential responses to presence and absence of a terminal amino group and a $\beta$-hydroxyl group. $A$, Responses to two basic AAs (orn and $l y s$ ) and their aliphatic counterparts (2-apa and 2-aha) are shown in the top row (color scale -0.5 to $7 \%$ ). Difference images are shown below (color scale $0-5 \%$ ). Note several categories of response properties (arrowhead, $\mathrm{nNH}_{2}$ required, chain length-independent; arrow, $\mathrm{nNH}_{2}$ required, chain lengthdependent; open arrowhead, $\mathrm{nNH}_{2}$ not tolerated, chain length-independent; open arrow, $\mathrm{nNH}_{2}$ not tolerated, chain length-dependent). Glomeruli activated by any compound ( $\mathrm{nNH}_{2}$ tolerated) are only visible in the top row, not in the difference images. $B$, Responses to two polar AAs (ser and thr) and their aliphatic counterparts (ala and 2-aba) are shown in the top row (color scale as above). Difference images are shown below. Several glomeruli do not tolerate the $\beta$-OH group; some of these are chain length-dependent (arrowhead). No glomeruli requiring the $\beta$-OH group are visible. Scale bar, $100 \mu \mathrm{m}$.

support for the validity of our image subtraction analysis and the conclusions drawn from it. The close correspondence of patterns obtained by cross-adaptation and subtraction analysis indicates that, indeed, an array of ORs with diverse tuning curves is involved in the response to even a simple odorant like an amino acid.

\section{DISCUSSION}

Neuronal representation of odorants is generally achieved via combinatorial activation of several broadly tuned glomeruli (Friedrich and Korsching, 1997; Galizia et al., 1999; Johnson et al., 1999; Rubin and Katz, 1999; Meister and Bonhoeffer, 2001). Recent molecular studies have shown a one-to-one correspondence of ORs and glomeruli such that terminals of ORNs expressing a single type of OR converge onto a single glomerulus (two for rodents) in the olfactory bulb or antennal lobe (Ressler et al., 1994; Mombaerts et al., 1996; Vosshall et al., 2000). Taking advantage of this OR-to-glomerulus correspondence, we have imaged presynaptic calcium changes in glomeruli, induced by a group of simple and systematically related odorants, to study odorant feature detection by a complete OR repertoire. Our approach complements the functional analysis of recombinant ORs (Krautwurst et al., 1998; Zhao et al., 1998; Malnic et al., 1999; Touhara et al., 1999; Araneda et al., 2000), which may not necessarily detect the main ligands of the ORs and would be practically impossible to use for the complete receptor repertoire.

The small size and transparent nature of the olfactory bulb in zebrafish are very convenient for imaging (Friedrich and Korsching, 1997) and allow visualization of all glomerular responses to an odorant. It should be noted that a glomerulus both integrates and averages signals of individual ORNs, and thus odor responses are expected to be somewhat more robust than those of individual ORNs. Because the calcium dye is introduced specifically in the terminals of the ORNs, glomerular signals should reflect the underlying receptor events of these cells and not contain intense bulbar processing [albeit some evidence for dopaminergic and GABAergic modulation has been uncovered recently (Keller et al., 1998; Koster et al., 1999; Aroniadou-Anderjaska et al., 2000)]. Other methods of imaging neuronal activity (e.g., 2-deoxyglucoseuptake, c-fos levels, intrinsic signals) do not distinguish between the input and output compartments of the glomeruli (Lancet et al., 1982; Guthrie et al., 1993; Cinelli et al., 1995; Johnson et al., 


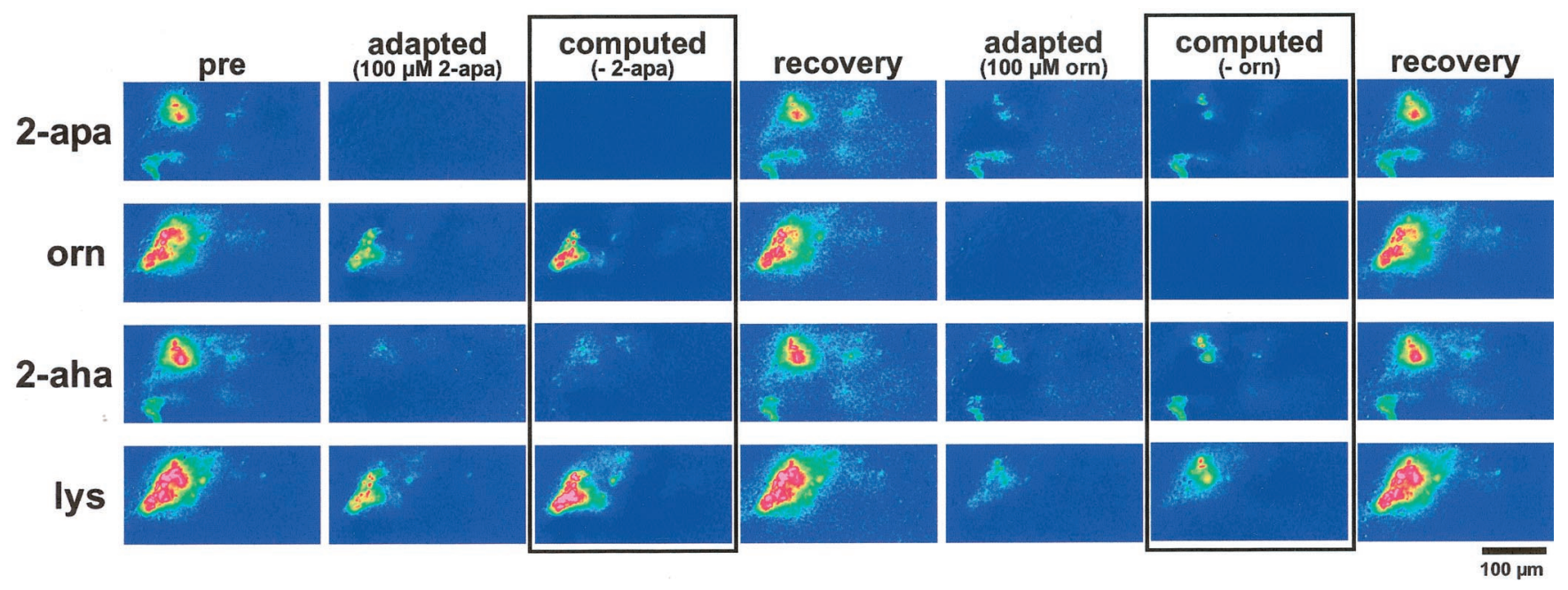

Figure 9. Adaptation and cross-adaptation of AA responses. Top to bottom, Responses to 2-apa, orn, 2-aha, and lys (color scale -0.5 to 5\%). Left to right, Consecutive stimuli are given: stimulus itself; adapted with 2-apa; encased, computed difference image (stimulus minus 2-apa response); stimulus itself after recovery period; adapted with orn; encased, computed difference image (stimulus minus orn response); stimulus itself after recovery period. Adaptation with stimulus leads to complete loss of response; cross-adaptation eliminates responses in a subset of glomeruli. Reversal of adaptation is complete ( $D I$ for preadaptation/postadaptation comparison in the range of intertrial $D I$ s). Difference images obtained by pixel-wise subtraction mimic adapted responses closely (color scale $0-3 \%$ ). The somewhat higher noise in the "biological subtraction" is characteristic for single trials. Images for the "digital subtraction" were averaged over four trials and accordingly show less noise. Scale bar, $100 \mu \mathrm{m}$.

1998, 1999; Yang et al., 1998; Galizia et al., 1999; Rubin and Katz, 1999; Johnson and Leon, 2000; Meister and Bonhoeffer, 2001).

Because the ORN-to-glomerulus correspondence has not been demonstrated in zebrafish so far, tuning properties of glomeruli may be broader than those of the underlying ORN, but in any case yield a minimal estimate for the ORN and thus OR specificity. The number of glomeruli with different tuning properties yields an estimate for the number of different ORs involved in the response to the corresponding odorants. The actual OR number might be higher, because testing of other analogs might have revealed differential sensitivities not detectable with the set of analogs examined here. Overestimation of OR numbers appears less likely. Mixed innervation of one glomerulus would result in a new, artificially broad response category, but cross-adaptation should only be partial in this case. We found cross-adaptation for broadly tuned glomeruli, i.e., no evidence for mixing of glomerular input.

Without exception, AA-responsive ORs require both the $\alpha$-amino and the $\alpha$-carboxyl group in the L-conformation. Electroolfactogram recording in catfish shows some response to D-ala (Caprio and Byrd, 1984). However, it is possible that this signal arises from a non-neuronal component of the electro-olfactogram like that observed for bile acids (Erickson and Caprio, 1984). A response domain requiring particular functional groups has been described in the rodent olfactory bulb as well [fatty acid/aldehyde domain (Imamura et al., 1992; Johnson and Leon, 2000; Uchida et al., 2000)]. Such a clustering of ORs that require a particular functional group may emerge as a general scheme for odorant representation, notwithstanding the differences in odorants between air-breathing and water-living taxa.

Requirements for the side chain are more relaxed, and different side chains recruit varying subsets of AA receptors. The structure of an AA odorant can therefore be divided into two subregions, the AA functional group and the side chain. The side chain consists of a hydrogen in the smallest AA possible, glycine. Even glycine was able to activate as many as 25 different glomeruli, but possibly fewer than 25 ORs, because some glomeruli might be innervated by ORNs expressing the same ORs. On the other hand, the actual number of glycine-responsive ORs should be larger than one, because the glycine response comprises foci with different dose-response curves. Thus, even the simplest AA is not represented by a single "glycine OR" but by a combination of "AAs-ORs," an impressive demonstration of the combinatorial nature of the odor code. Successive elongation of the side chain resulted in the recruitment of additional glomeruli and therefore additional ORs. A lower-bound estimate of at least five different ORs involved in the response to neutral aliphatic AAs can be derived from the number of different minimal chain-length requirements of glomeruli.

Most glomeruli show a continuous increase of signal strength with increasing chain length. Only a few glomeruli were found to be inversely tuned, preferentially to shorter chain lengths. It is remarkable that these tuning properties are matched closely by the representation of fatty acids in the rat olfactory bulb as studied by 2-deoxyglucose labeling (Johnson et al., 1999), and they are also observed for isolated mammalian ORNs (Malnic et al., 1999). Conflicting results (bell-shaped tuning curves) could be caused by difficulties in obtaining identical vapor concentrations for different chemicals (Rubin and Katz, 1999; Araneda et al., 2000; Meister and Bonhoeffer, 2001), a problem not present for water-soluble odorants. Other properties of response patterns and tuning curves for volatile odorants, like the increasing complexity of the response pattern with increasing chain length and definite pattern changes for small increases in chain length (Johnson et al., 1998, 1999; Uchida et al., 2000; Meister and Bonhoeffer, 2001), are also similar to results reported here for a group of water-soluble odorants (AAs). However, it is difficult to interpret these similarities, one reason being that none of those studies has measured specifically the receptor neuron component of the glomerular signal. To distinguish among contributions from receptor neuron input, mitral cell output, and activity from inhibitory interneurons, it will be necessary to develop specific imaging methods for the postsynaptic compartment in the olfactory bulb, the mitral cells, to complement existing electrophysiological stud- 
Figure 10. A model for the binding sites of AA receptors. AA ligand/AA receptor interactions are shown according to the categories found in the difference analysis. A selection of receptors (rounded rectangles) is shown in the top row. The bottom row lists a reduced set of ligands (black) and nonligands (gray) below the corresponding receptor. Representatives of the different AA odorant classes are shown (short-chain neutral: alanine; long-chain neutral: 2-aha; basic: lysine; polar: serine). The ligand depicted is underlined. Mandatory binding sites are indicated by horizontal dashes; facultative interactions (not required but not destructive either) are indicated by stippling. The binding site for the AA head (black) is mandatory for all AA receptors. The amount of required interaction with the carbon side chain (dark gray) varies (compare $O R 1$ with $O R 2$ ), but chain lengths
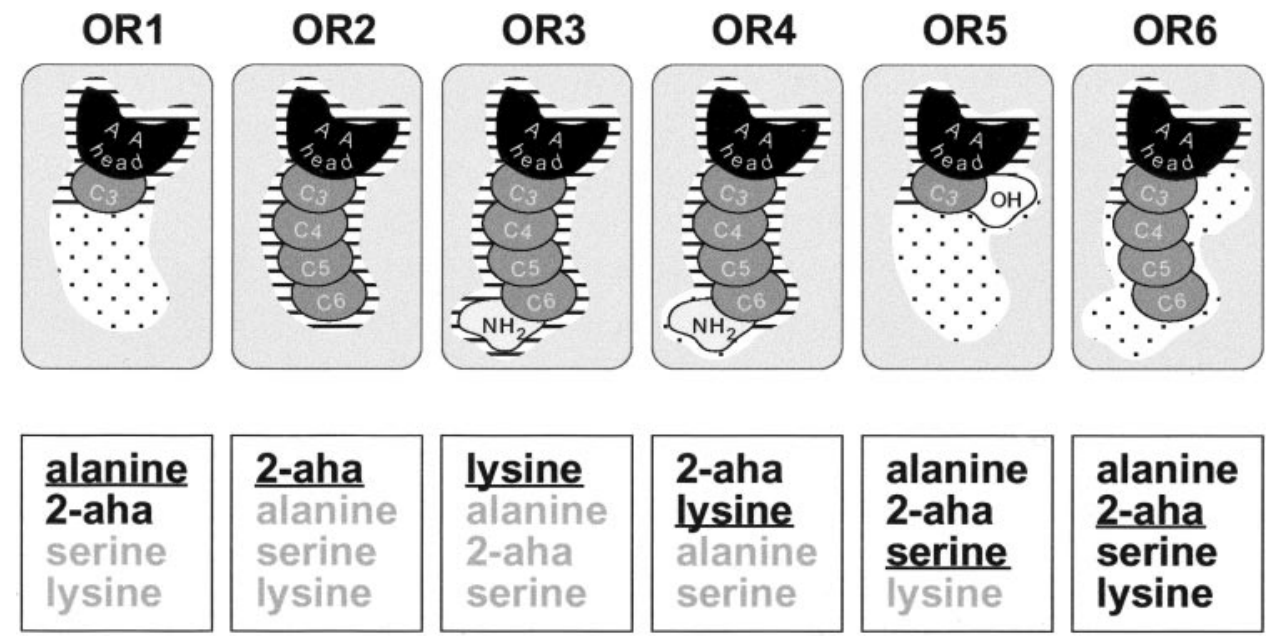

beyond the requirements are always tolerated (OR1). The terminal amino group (light gray) binding site is mandatory in OR 3 but facultative in OR4 and OR6. The hydroxy side chain (white) is never required, but is tolerated in OR5 and OR6.

ies (Imamura et al., 1992; Mori et al., 1992; Katoh et al., 1993; Yokoi et al., 1995).

Concentration is another parameter that heavily influences the response pattern. Presumably because of the affinity differences within the receptor repertoire recruited to a response, pattern dissimilarities for responses to different concentrations of the same compound are pronounced. It should be noted that some combinations of different chain length and different concentration elicit patterns more similar to each other than the corresponding uni-dimensional change in concentration. In these cases, however, pattern dissimilarity is mostly reduced but not eliminated. This is consistent with the notion that odor stimuli might be discriminated from each other regardless of their concentration.

The introduction of amino and hydroxyl functional groups in the side chain visualized further subdivisions of the receptor repertoire and thus indicates the presence of additional receptors within the group of AA-responsive ORs. Interestingly, no receptors specific for the polar AAs serine and threonine were found; i.e., the $\beta$-hydroxyl group does not seem to participate in the binding interaction.

On the basis of our results, we propose a model for the AA-OR interaction (Fig. 10). AA structural motives may be required, tolerated, or destructive for the binding interaction. A mandatory binding site for the AA head comprises the $\alpha$-amino group and the $\alpha$-carboxyl group in the L-conformation. An additional binding site serves as detector of minimal side-chain length (absent in glycine-responsive ORs). Some ORs possess another binding site that requires a terminal amino group in the context of an $\alpha$-AA side chain. Furthermore, larger or smaller "tolerance regions" appear to be present that admit, but do not require, a range of different side chains (length and functional group differences). The $\alpha$-hydrogen seems to lie also at a position of limited tolerance (Hara, 1976, 1977). Thus, individual receptors of the AA receptor family differ within at least one or two structural binding motives beyond the AA configuration per se and differ also in the degree of tolerance afforded by the binding pocket. These data suggest that ORs recognize a combination of molecular features (odotopes), not single odotopes.

The combination of ORs activated by a particular odorant represents the "finger print" of that odorant. In this finger print, the absence of response of particular ORs is as meaningful and distinctive as the presence of response of other ORs. Presumably, not the absolute levels of receptor activation but their ratios determine the perception of odor quality, analogous to the situation in color vision (Bartels and Zeki, 2000). The dissimilarity indices that we introduced normalize for intensity; i.e., they measure differences in ratios of receptor activation. Dissimilarity indices even for closely related stimuli significantly above those observed for repeated stimulation with the same odorant ascertain the high discriminatory power of the zebrafish OR repertoire. Well above 10 differently tuned ORs (Table 1) appear to generate the observed discriminating abilities. This estimate extends earlier lower-bound estimates of at least four different ORs (Caprio and Byrd, 1984; Friedrich and Korsching, 1997). Thus a considerable proportion of all ORs [estimated to be $\sim 100$ (Ngai et al., 1993; Weth et al., 1996)] seems devoted to processing AA odors.

Odor space is not restricted to naturally occurring stimuli. "Unphysiological" AAs recruit more glomeruli than the proteinogenic AAs tested. Thus, ORs do not seem to be selected for odorants encountered in the normal environment. On the other hand, ORs have not evolved for all potential odorants. AArelated compounds such as fatty acids and amines do not elicit responses in the fish olfactory system, although both are potent odorants for mammals. Such restrictions of the fish odor space may reflect the behavioral relevance of the corresponding chemical groups of stimuli. AAs indicate food sources, and many AAs can be distinguished by catfish, some by goldfish, a close relative of zebrafish, in behavioral studies (Zippel et al., 1993; Valentincic et al., 2000). However, further investigation will be necessary to determine whether the astonishingly high resolving power for minutely different chemical structures and even different concentrations of the same stimulus (measured at the glomerular level and inferred for the underlying ORs) may be present at all later stages of olfactory information processing and, ultimately, at the behavioral level.

\section{REFERENCES}

Araneda R, Kini A, Firestein S (2000) The molecular receptive range of an odorant receptor. Nat Neurosci 3:1248-1255.

Aroniadou-Anderjaska V, Zhou FM, Priest CA, Ennis M, Shipley MT (2000) Tonic and synaptically evoked presynaptic inhibition of sensory input to the rat olfactory bulb via GABA(B) heteroreceptors. J Neurophysiol 84:1194-1203.

Bartels A, Zeki S (2000) The architecture of the colour centre in the 
human visual brain: new results and a review. Eur J Neurosci 12:172-193.

Barth AL, Justice NJ, Ngai J (1996) Asynchronous onset of odorant receptor expression in the developing zebrafish olfactory system. Neuron 16:23-34.

Buck L, Axel R (1991) A novel multigene family may encode odorant receptors: a molecular basis for odor recognition. Cell 65:175-187.

Caprio J, Byrd RP (1984) Electrophysiological evidence for acidic, basic, and neutral amino acid olfactory receptor sites in the catfish. J Gen Physiol 84:403-422.

Cinelli AR, Hamilton KA, Kauer JS (1995) Salamander olfactory bulb neuronal activity observed by video rate, voltage-sensitive dye imaging. 3. Spatial and temporal properties of responses evoked by odorant stimulation. J Neurophysiol 73:2053-2071.

Erickson JR, Caprio J (1984) The spatial distribution of ciliated and microvillous olfactory receptor neurons in the channel catfish is not matched by a differential specificity to amino acids and bile salt stimuli. Chem Senses 9:127-141.

Friedrich RW, Korsching SI (1997) Combinatorial and chemotopic odorant coding in the zebrafish olfactory bulb visualized by optical imaging. Neuron 18:737-752.

Friedrich RW, Korsching SI (1998) Chemotopic, combinatorial, and noncombinatorial odorant representations in the olfactory bulb revealed using a voltage-sensitive axon tracer. J Neurosci 18:9977-9988.

Galizia CG, Sachse S, Rappert A, Menzel R (1999) The glomerular code for odor representation is species specific in the honeybee Apis mellifera. Nat Neurosci 2:473-478.

Guthrie KM, Anderson AJ, Leon M, Gall C (1993) Odor-induced increases in c-fos mRNA expression reveal an anatomical "unit" for odor processing in olfactory bulb. Proc Natl Acad Sci USA 90:3329-3333.

Hara TJ (1976) Structure-activity relationships of amino acids in fish olfaction. Comp Biochem Physiol A Physiol 54:31-36.

Hara TJ (1977) Further studies on the structure-activity relationships of amino acids in fish olfaction. Comp Biochem Physiol A Physiol 56:559-565.

Imamura K, Mataga N, Mori K (1992) Coding of odor molecules by mitral/tufted cells in rabbit olfactory bulb. I. Aliphatic compounds. J Neurophysiol 68:1986-2002.

Johnson BA, Leon M (2000) Modular representations of odorants in the glomerular layer of the rat olfactory bulb and the effects of stimulus concentration. J Comp Neurol 422:496-509.

Johnson BA, Woo CC, Leon M (1998) Spatial coding of odorant features in the glomerular layer of the rat olfactory bulb. J Comp Neurol 393:457-471.

Johnson BA, Woo CC, Hingco EE, Pham KL, Leon M (1999) Multidimensional chemotopic responses to n-aliphatic acid odorants in the rat olfactory bulb. J Comp Neurol 409:529-548.

Katoh K, Koshimoto H, Tani A, Mori K (1993) Coding of odor molecules by mitral/tufted cells in rabbit olfactory bulb. II. Aromatic compounds. J Neurophysiol 70:2161-2175.

Keller A, Yagodin S, Aroniadouanderjaska V, Zimmer LA, Ennis M, Sheppard NF, Shipley MT (1998) Functional organization of rat olfactory bulb glomeruli revealed by optical imaging. J Neurosci 18:2602-2612.

Korsching SI, Argo S, Campenhausen H, Friedrich RW, Rummrich A, Weth F (1997) Olfaction in zebrafish: what does a tiny teleost tell us. Semin Cell Dev Biol 8:181-187.

Koster NL, Norman AB, Richtand NM, Nickell WT, Puche AC, Pixley SK, Shipley MT (1999) Olfactory receptor neurons express D2 dopamine receptors. J Comp Neurol 411:666-673.

Krautwurst D, Yau KW, Reed RR (1998) Identification of ligands for olfactory receptors by functional expression of a receptor library. Cell 95:917-926.

Lancet D, Greer CA, Kauer JS, Shepherd GM (1982) Mapping of odorrelated neuronal activity in the olfactory bulb by high-resolution 2-deoxyglucose autoradiography. Proc Natl Acad Sci USA 79:670-674.

Laska M, Teubner P (1998) Odor structure-activity relationships of carboxylic acids correspond between squirrel monkeys and humans. Am J Physiol 274:R1639-R1645

Malnic B, Hirono J, Sato T, Buck LB (1999) Combinatorial receptor codes for odors. Cell 96:713-723.

Mathieson WB, Maler L (1988) Morphological and electrophysiological properties of a novel in vitro preparation: the electrosensory lateral line lobe brain slice. J Comp Physiol [A] 163:489-506.

Meister M, Bonhoeffer T (2001) Tuning and topography in an odor map on the rat olfactory bulb. J Neurosci 21:1351-1360.
Mombaerts P, Wang F, Dulac C, Chao SK, Nemes A, Mendelsohn M, Edmondson J, Axel R (1996) Visualizing an olfactory sensory map. Cell 87:675-686.

Mori K, Mataga N, Imamura K (1992) Differential specificities of single mitral cells in rabbit olfactory bulb for a homologous series of fatty acid odor molecules. J Neurophysiol 67:786-789.

Mori K, Nagao H, Yoshihara Y (1999) The olfactory bulb: coding and processing of odor molecule information. Science 286:711-715.

Ngai J, Dowling MM, Buck L, Axel R, Chess A (1993) The family of genes encoding odorant receptors in the channel catfish. Cell 72:657-666

Rawson NE, Eberwine J, Dotson R, Jackson J, Ulrich P, Restrepo D (2000) Expression of mRNAs encoding for two different olfactory receptors in a subset of olfactory receptor neurons. J Neurochem 75:185-195.

Ressler KJ, Sullivan SL, Buck LB (1994) Information coding in the olfactory system: evidence for a stereotyped and highly organized epitope map in the olfactory bulb. Cell 79:1245-1255.

Rubin BD, Katz LC (1999) Optical imaging of odorant representations in the mammalian olfactory bulb. Neuron 23:499-511.

Shepherd GM (1993) Principles of specificity and redundancy underlying the organization of the olfactory system. Microsc Res Tech 24:106-112

Speca DJ, Lin DM, Sorensen PW, Isacoff EY, Ngai J, Dittman AH (1999) Functional identification of a goldfish odorant receptor. Neuron 23:487-498.

Strotmann J, Conzelmann S, Beck A, Feinstein P, Breer H, Mombaerts P (2000) Local permutations in the glomerular array of the mouse olfactory bulb. J Neurosci 20:6927-6938.

Sveinsson T, Hara TJ (1990) Analysis of olfactory responses to amino acids in arctic char (Salvelinus alpinus) using a linear multiple-receptor model. Comp Biochem Physiol A Physiol 97:279-287.

Touhara K, Sengoku S, Inaki K, Tsuboi A, Hirono J, Sato T, Sakano H, Haga T (1999) Functional identification and reconstitution of an odorant receptor in single olfactory neurons. Proc Natl Acad Sci USA 96:4040-4045.

Troemel ER, Chou JH, Dwyer ND, Colbert HA, Bargmann CI (1995) Divergent seven transmembrane receptors are candidate chemosensory receptors in C. elegans. Cell 83:207-218.

Tsuboi A, Yoshihara S, Yamazaki N, Kasai H, Asai-Tsuboi H, Komatsu M, Serizawa S, Ishii T, Matsuda Y, Nagawa F, Sakano H (1999) Olfactory neurons expressing closely linked and homologous odorant receptor genes tend to project their axons to neighboring glomeruli on the olfactory bulb. J Neurosci 19:8409-8418.

Uchida N, Takahashi Y, Tanifuji M, Mori K (2000) Odor maps in the mammalian olfactory bulb: domain organization and odorant structural features. Nat Neurosci 3:1035-1043.

Valentincic T, Metelko J, Ota D, Pirc V, Blejec A (2000) Olfactory discrimination of amino acids in brown bullhead catfish. Chem Senses 25:21-29.

Vosshall L, Wong A, Axel R (2000) An olfactory sensory map in the fly brain. Cell 102:147-159.

Wang F, Nemes A, Mendelsohn M, Axel R (1998) Odorant receptors govern the formation of a precise topographic map. Cell 93:47-60.

Weth F, Nadler W, Korsching S (1996) Nested expression domains for odorant receptors in zebrafish olfactory epithelium. Proc Natl Acad Sci USA 93:13321-13326.

Wetzel CH, Oles M, Wellerdieck C, Kuczkowiak M, Gisselmann G, Hatt H (1999) Specificity and sensitivity of a human olfactory receptor functionally expressed in human embryonic kidney 293 cells and $X e$ nopus laevis oocytes. J Neurosci 19:7426-7433.

Yang X, Renken R, Hyder F, Siddeek M, Greer CA, Shepherd GM, Shulman RG (1998) Dynamic mapping at the laminar level of odorelicited responses in rat olfactory bulb by functional MRI. Proc Natl Acad Sci USA 95:7715-7720.

Yokoi M, Mori K, Nakanishi S (1995) Refinement of odor molecule tuning by dendrodendritic synaptic inhibition in the olfactory bulb. Proc Natl Acad Sci USA 92:3371-3375.

Zhao H, Ivic L, Otaki JM, Hashimoto M, Mikoshiba K, Firestein S (1998) Functional expression of a mammalian odorant receptor. Science 279:237-242.

Zippel HP, Voigt R, Knaust M, Luan Y (1993) Spontaneous behaviour, training and discrimination training in goldfish using chemosensory stimuli. J Comp Physiol [A] 172:81-90. 